\title{
Terahertz electromodulation spectroscopy of electron transport in GaN
}

\author{
S. G. Engelbrecht, ${ }^{1}$ T. R. Arend, ${ }^{1}$ T. Zhu, ${ }^{2}$ M. J. Kappers, ${ }^{2}$ and R. Kersting ${ }^{1, a)}$ \\ 1) Photonics and Optoelectronics Group, Physics Department and Center for NanoScience (CeNS), \\ Ludwig-Maximilians-Universität, Amalienstr. 54, 80799 München, Germany \\ ${ }^{2)}$ Department of Materials Science and Metallurgy, University of Cambridge, \\ Cambridge CB3 OFS
}

(Dated: 2 February 2015)

Time-resolved terahertz $(\mathrm{THz})$ electromodulation spectroscopy is applied to investigate the high-frequency transport of electrons in gallium nitride at different doping concentrations and densities of threading dislocations. At $\mathrm{THz}$ frequencies, all structures reveal Drude transport. The analysis of the spectral response provides the fundamental transport properties, such as the electron scattering time and the electrons' conductivity effective mass. We observe the expected impact of ionized-impurity scattering and that scattering at threading dislocations only marginally affects the high-frequency mobility.

PACS numbers: 71.55.Eq, 73.40.Qv, 72.10.Fk, 72.20.-i

Keywords: gallium nitride, terahertz spectroscopy, electrical conductivity

The Drude model of electrical transport holds for remarkably many solid state materials although it does not consider the quantum mechanical background of electronic transport ${ }^{1,2}$. During the past decade, terahertz $(\mathrm{THz})$ spectroscopy has become a suitable characterization tool for measuring high-frequency transport properties. Many studies that followed the pioneering works ${ }^{3,4}$ verified Drude-like transport at $\mathrm{THz}$ frequencies for most crystalline semiconductors. The high-frequency transport in GaN, however, was interpreted using a variety of models. In unintentionally doped GaN layers, a pure Drude response was found ${ }^{5}$. Other studies reported deviations from Drude behavior and described them with the Kohlrausch stretched exponential model ${ }^{6}$ or with a combination of a Drude response and a classical damped oscillator model ${ }^{7}$. Transport in InGaN/GaN quantum wells was reproduced using the Drude-Smith model ${ }^{8}$, and plasmon resonances were observed in grating-gate GaN transistor structures ${ }^{9}$.

In this work, we present a study on epitaxial GaN layers using time-resolved $\mathrm{THz}$ electromodulation spectroscopy. Recently, we showed that the technique yields fundamental transport properties, such as the Drude scattering time $\tau$, as well as the conductivity effective mass $m_{c}^{*}$. Terahertz electromodulation spectroscopy can be widely applied, as illustrated by work on $\mathrm{GaAs}^{10}$, silicon ${ }^{11}$, nanocrystalline $\mathrm{ZnO}^{12}$, and pentacene ${ }^{13}$. In this work, the high-frequency response of electrons in GaN is investigated in devices grown by metal organic chemical vapor phase epitaxy (MOVPE) on sapphire. At high doping densities $\left(n_{3 D}>10^{18} \mathrm{~cm}^{-3}\right)$, THz spectroscopy and conventional electronic characterization yield nearly identical mobilities. At low densities, two obstacles make the electronic characterization challenging: i) the quality of the ohmic contacts to the electron gas at the surface and ii) parallel conducting paths

a) Electronic mail: roland.kersting@lmu.de close to the interface between GaN and sapphire. We show that even at low doping densities where classical methods fail, THz spectroscopy provides insight into the fundamental transport properties.

Three GaN structures were grown by MOVPE in a 6x2" Thomas Swan close-coupled showerhead reactor. The details of the sample growth methods can be found elsewhere $^{14,15}$. The GaN samples differ in threading dislocation density (TDD), as well as in the intentional silicon dopant concentration to achieve n-type conductivity in the GaN capping layer. The TDD was either $4 \times 10^{8}$ $\mathrm{cm}^{-2}$ (samples 1 and 3 ) or $4 \times 10^{9} \mathrm{~cm}^{-2}$ (sample 2) as determined by atomic force microscopy on similarly grown samples ${ }^{16}$. The Si-doping concentration was varied to give an electron density of $5 \times 10^{16} \mathrm{~cm}^{-3}$ (samples 1 and 2 ) or $3.5 \times 10^{18} \mathrm{~cm}^{-3}$ (sample 3 ) as measured by mercuryprobe capacitance-voltage or Hall measurements. Table I summarizes the samples' parameters. The low doping concentrations of devices 1 and 2 cause inferior ohmic contacts, which limits Hall measurements. Therefore, only the mobility obtained on device 3 appears realistic.

\begin{tabular}{l|c|c|c} 
& $\mathrm{TDD}\left(\mathrm{cm}^{-2}\right)$ & $n_{3 D}\left(\mathrm{~cm}^{-3}\right)$ & $\mu\left(\mathrm{cm}^{2} / \mathrm{Vs}\right)$ \\
\hline Device 1 & $4 \times 10^{8}$ & $5 \times 10^{16}$ & N/A \\
Device 2 & $4 \times 10^{9}$ & $5 \times 10^{16}$ & 92 \\
Device 3 & $4 \times 10^{8}$ & $3.5 \times 10^{18}$ & 254
\end{tabular}

TABLE I. Properties of the Si-doped top layer in the three GaN structures, such as threading dislocation density (TDD), carrier density $n_{3 D}$, determined by mercury-probe CV or Hall measurements, and carrier mobility as determined by Hall characterization.

As indicated in the diagram of Fig. 1, the devices 1 and 3 contain an unintentionally oxygen-doped region of GaN near the sapphire substrate as a result of their growth procedure ${ }^{14}$. This highly compensated n-type region is characterized by a high sheet carrier density and low mobility depending on the growth method ${ }^{17,18}$, typically $n_{2 D} \approx 2 \times 10^{14} \mathrm{~cm}^{-2}$ and $\mu \approx 150 \mathrm{~cm}^{2} / \mathrm{Vs}$ 
for our unintentionally doped GaN layers with a TDD of $4 \times 10^{8} \mathrm{~cm}^{-2}$. A reliable mobility value for device 1 (see Table I) could not be obtained from standard Hall measurements as the Hall sheet carrier density of device 1 was measured at $8 \times 10^{13} \mathrm{~cm}^{-2}$, greatly exceeding that of the Si-doped top layer as measured by Hg-probe $\left(5 \times 10^{12} \mathrm{~cm}^{-2}\right)$, making a correction using a two-layer model unreliable. Due to a different growth procedure, the degenerate interface region is absent in device 2 (at the expense of a higher TDD) and the carrier concentration measured by Hall-effect and mercuryprobe are the same. It is noted that the Hall mobility values measured for Si-doped GaN layers of the same type as device 2 grown in our lab over the range from $n_{3 D}=2 \times 10^{17}$ to $2 \times 10^{19} \mathrm{~cm}^{-3}$ equal theoretical mobility values ${ }^{19}$ when a compensation ratio $\left(C=N_{A} / N_{D}\right)$ of 0.4 is applied. Below that range of $n_{3 D}$, as for device 2 , the Hall mobility is significantly reduced due to scattering processes at charged threading dislocations ${ }^{20}$. Finally, the Hall electron density for device 3 matches the value from the mercury-probe measurements after correction for the parallel conduction at the interface using a two-layer analysis, while its mobility value is typical for $C=0.4$.

Terahertz characterization by electromodulation spectroscopy requires switching the electron sheet density $n_{2 D}$ within the semiconductor. This is achieved with Schottky devices as illustrated in Fig. 1. For the ohmic contacts to the GaN, we alloyed pads of $15 \mathrm{~nm} \mathrm{Ti,} 115 \mathrm{~nm}$ $\mathrm{Al}$, and $40 \mathrm{~nm} \mathrm{Au}$ at $500^{\circ} \mathrm{C}$ in $\mathrm{Ar} / \mathrm{H}_{2}$ atmosphere ${ }^{21}$. An insulating layer of $450 \mathrm{~nm}$ of parylene- $\mathrm{N}$ is de-

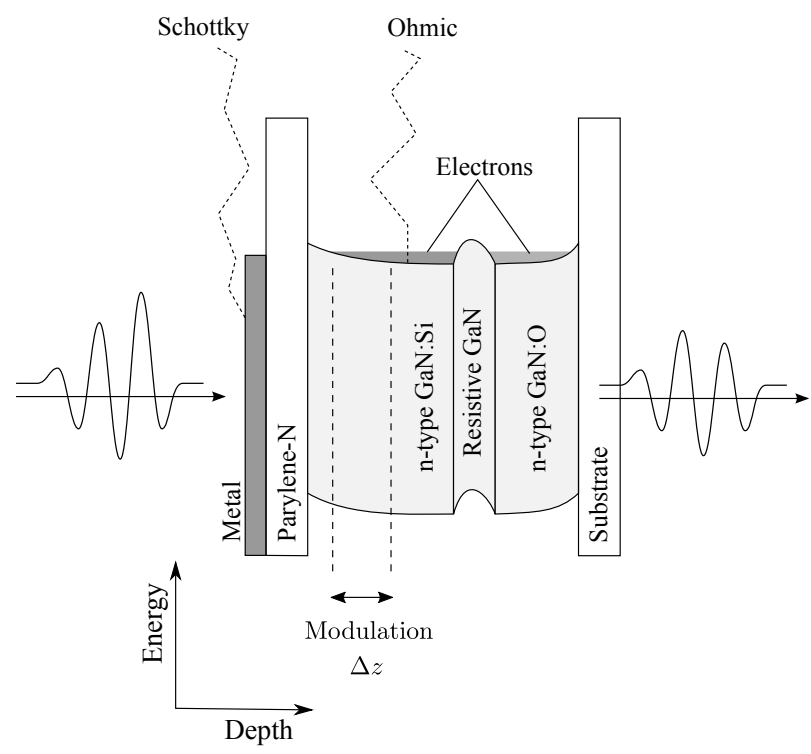

FIG. 1. Schematic band diagram of devices 1 and 3 in equilibrium. The layer sequence of device 2 has no GaN:O layer. The sheet density of the electrons is modulated by the bias applied to the Schottky contact. The modulation affects absorption and dispersion of the $\mathrm{THz}$ pulses transmitted through the device. posited directly onto the GaN surface by chemical vapor deposition $^{22}$. The top layer consists of $7 \mathrm{~nm}$ chromium, has a diameter of $5 \mathrm{~mm}$, and serves as Schottky contact. Application of a negative bias at the Schottky contact depletes the GaN from electrons at the end of the depletion zone. Typical changes in the sheet carrier concentration are $\Delta n_{2 D} \approx 8 \times 10^{11} \mathrm{~cm}^{-2}$.

The THz experiments are performed using a setup similar to most $\mathrm{THz}$ time-domain spectrometers. Few-cycle $\mathrm{THz}$ pulses are generated by femtosecond laser excitation of an interdigitated emitter structure at a repetition rate of $80 \mathrm{MHz}$. After transmission through the device, the $\mathrm{THz}$ radiation is time-resolved by electro-optic sampling in a $1 \mathrm{~mm}$ thick ZnTe crystal. The accessible frequency range extends from about $0.2 \mathrm{THz}$ to $2.8 \mathrm{THz}$. The center peak of the pulse has a field strength of about $60 \mathrm{kV} / \mathrm{cm}$, and the signal-to-noise ratio of the setup exceeds $3.5 \times 10^{5} \mathrm{~Hz}^{1 / 2}$ when the chamber is pumped down to 0.02 mbar. Further technical details are published elsewhere $^{10,12}$.

When $\mathrm{THz}$ radiation is transmitted through the GaN devices, every layer contributes to the overall dispersion and absorption of the $\mathrm{THz}$ pulses. Electromodulation experiments allow for extracting the electronic response of the charge carriers by switching the state of the car-

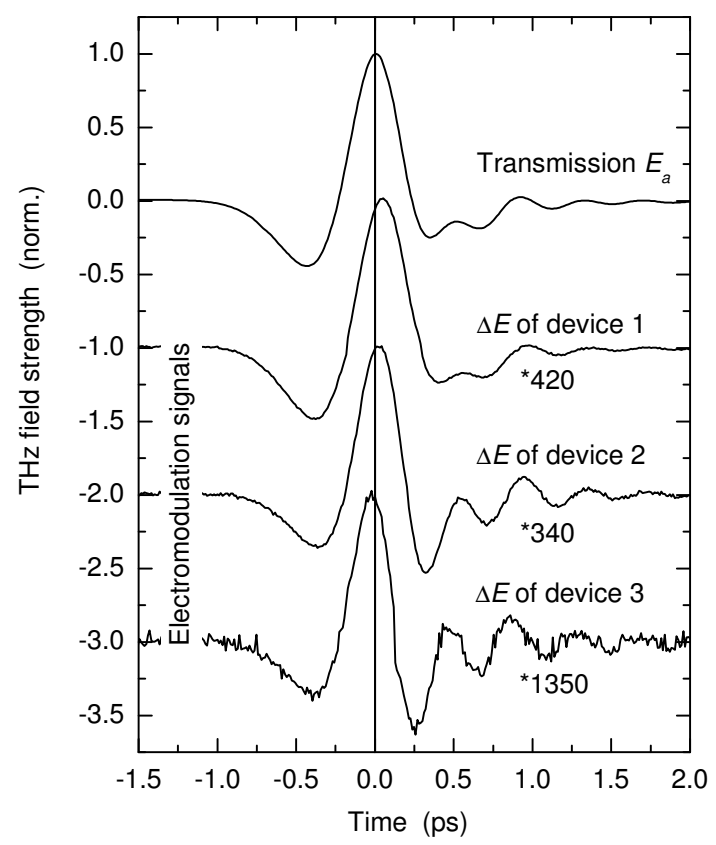

FIG. 2. Time-resolved field $E_{a}(t)$ after transmission through device 1 and modulation signals $\Delta E(t)$ for devices 1,2 , and 3. Transient transmission signals for devices 2 and 3 are not shown, because they are similar to $E_{a}(t)$ recorded on device 1 . The modulation signals are offset for clarity. All modulation signals are recorded by depleting the devices. Experiments performed by accumulating electrons in the GaN yield nearly identical results. 
Device 1
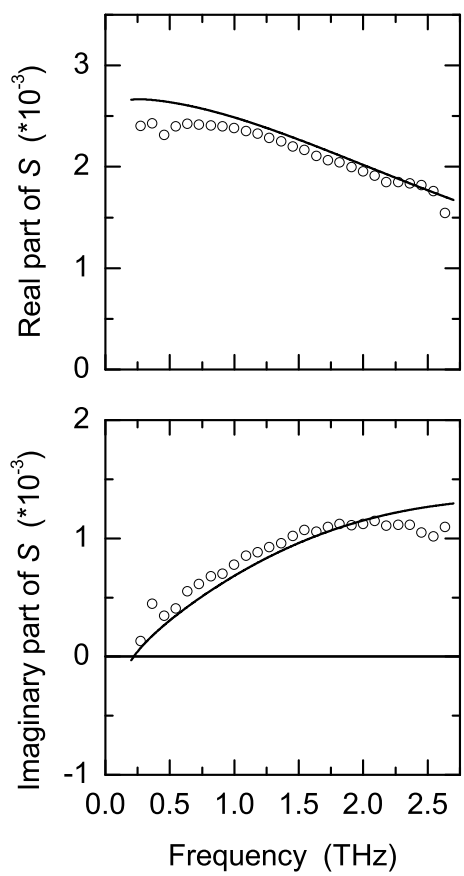

Device 2
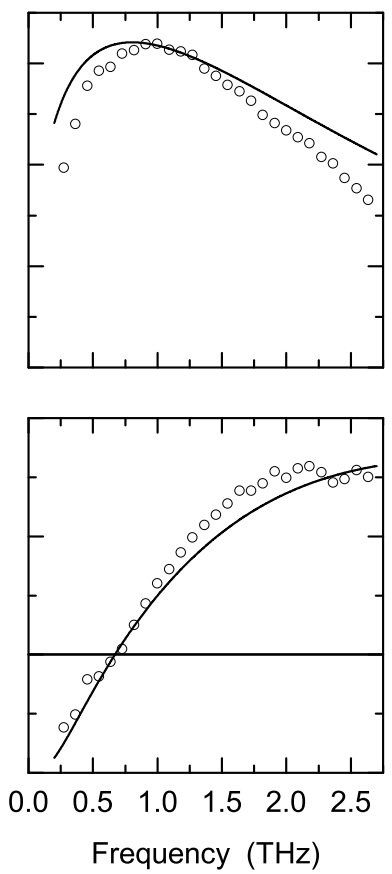

Device 3
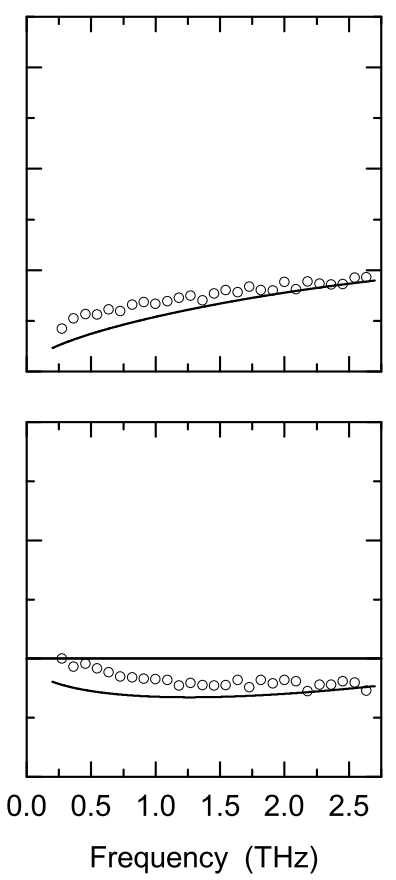

FIG. 3. Frequency dependence of the real part and the imaginary part of the relative differential signal $S(\omega)$ recorded on samples 1 to 3 (symbols). The solid lines are fits using the Drude model. The confidence interval between 0.5 and $2.2 \mathrm{THz}$ is approximately the size of the circles, which illustrate the experimental data.

rier distributions from equilibrium to depletion, accumulation, or inversion ${ }^{11}$. In our experiments, we record the transmitted fields $E_{a}(t)$ and $E_{b}(t)$ of the unbiased device and the biased device, respectively. Switching the sheet carrier density by $\Delta n_{2 D}$ at the end of the depletion zone causes a differential $\mathrm{THz}$ transmission $\Delta E(t)=E_{b}(t)-E_{a}(t)$. Figure 2 shows the transmitted field $E_{a}(t)$, as well as the differential signal $\Delta E(t)$, when depleting the electron layer partially. For all devices, the differential signals are orders of magnitude smaller than $E_{a}(t)$. On first glance, the transients $E_{a}(t)$ and $\Delta E(t)$ appear similar. The center peaks, however, indicate a minute phase shift in $\Delta E(t)$. In the case of device 1 , this phase delay is positive, which is in agreement with the picture of an electron gas that lags behind the driving field. Device 2 shows a reduced phase lag, and for device 3 , the phase even gets negative.

Fourier transforms of the relative modulation signals $S(\omega)=\Delta E(\omega) / E(\omega)$ are shown in Fig. 3. The modulated carrier sheet densities were $7.4 \times 10^{11} \mathrm{~cm}^{-2}, 8.8 \times$ $10^{11} \mathrm{~cm}^{-2}$, and $7.6 \times 10^{11} \mathrm{~cm}^{-2}$, for devices 1,2 , and 3 , respectively. The precise deduction of $\Delta n_{2 D}$ from the load current of the device is crucial for the quantitative analysis of the $\mathrm{THz}$ data, and care is taken that statistical errors do not exceed $1 \%$.

The most extraordinary feature in Fig. 3 is that the imaginary part of $S(\omega)$ is partially negative for device 2 , and for device 3 , it is negative for the entire frequency range. This behavior is unexpected for a Drude response of a dilute carrier gas, as discussed in previous $\mathrm{THz}$ studies ${ }^{10,11,23}$. The frequency-dependent data are in accordance with the preceding differential signal in the time-domain, which was observed on device 3 (see Fig. 2). The negative imaginary part of $S$ also leads to a negative phase $\tan \phi=\operatorname{Im}(S) / \operatorname{Re}(S)$ and thus to a differential signal $\Delta E(t)$, which precedes the driving $\mathrm{THz}$ field $E_{a}(t)$.

The analysis of the frequency-domain data requires an approach that covers the response of dilute electron gases, as for instance in devices 1 and 2, as well as the dynamics of dense gases, which are much more opaque to $\mathrm{THz}$ radiation (device 3 ). Both situations can be treated by considering the dependence of the wavevector on the conductivity of the electron gas. In the conducting layers of the GaN, the wavevector of the propagating field is ${ }^{24}$ :

$$
k_{a}=\sqrt{\omega^{2} \epsilon_{r} \epsilon_{0} \mu_{0}+i \omega \mu_{0} \sigma(\omega)} \quad,
$$

where $\sigma(\omega)$ is the frequency-dependent conductivity of the carriers. The background permittivity of the GaN and the vacuum permeability are given by $\epsilon_{r} \epsilon_{0}$ and $\mu_{0}$, respectively. The modulation of the carrier sheet density 
by $\Delta n_{2 D}$ is accompanied by a change in the thickness of the conducting layer given by $\Delta z=\Delta n_{2 D} / n_{3 D}$. In the case of a depleted structure, the conductivity is zero along $\Delta z$, which reduces the wavevector given by eq. (1) from $k_{a}$ to $k_{b}=\sqrt{\omega^{2} \epsilon_{r} \epsilon_{0} \mu_{0}}$. The differential signal due to the modulated electron gas is:

$$
S(\omega)=\frac{E_{b}(\omega)-E_{a}(\omega)}{E_{a}(\omega)}=\frac{e^{i\left(k_{b} \Delta z-\omega t\right)}-e^{i\left(k_{a} \Delta z-\omega t\right)}}{e^{i\left(k_{a} \Delta z-\omega t\right)}}
$$

The same holds for experiments in which carriers are accumulated. For the calculation of $k_{a}$, the AC Drude conductivity is used

$$
\sigma(\omega)=\frac{n_{3 D} e^{2} \tau}{m_{c}^{*}} \cdot \frac{1+i \omega \tau}{1+\omega^{2} \tau^{2}}
$$

where $\tau$ is the Drude scattering time and $m_{c}^{*}$ the conductivity effective mass of the electrons. Treating the reflection at the interface between the insulating GaN and the conducting layer can be omitted because only the position of the interface is changed by the modulation.

The calculations shown in Fig. 3 reproduce the experimental data for all three devices. Fit parameters are the conductivity effective mass $m_{c}^{*}$, the Drude scattering time $\tau$ and the density $n_{3 D}$ of mobile electrons that contribute to the $\mathrm{THz}$ response. Table II summarizes the fundamental transport parameters deduced from the $\mathrm{THz}$ data and the Dude mobility calculated from $\mu=e \tau / m_{c}^{*}$. For all three devices, the same conductivity effective mass $m_{c}^{*}=0.22 m_{e}$ was found, which agrees with commonly used values ${ }^{25-27}$. The $\mathrm{THz}$ data also reproduce the doping densities in devices 2 and 3 . For device 1, however, the electron density extracted from the $\mathrm{THz}$ data is one order of magnitude smaller than the density obtained by capacitance-voltage characterization. One explanation is that in this device not all electrons introduced by doping contribute to the $\mathrm{THz}$ response. Previous studies have found activation energies of several $\mathrm{meV}$ in $\mathrm{GaN}^{17,18}$. This supports the explanation, because bound carriers respond to the $\mathrm{THz}$ field with negligible polarization.

\begin{tabular}{c|c|c|c|c} 
& $m_{c}^{*}\left(m_{e}\right)$ & $\tau(\mathrm{fs})$ & $n_{3 D}\left(\mathrm{~cm}^{-3}\right)$ & $\mu\left(\mathrm{cm}^{2} / \mathrm{Vs}\right)$ \\
\hline Device 1 & 0.22 & 46 & $5 \times 10^{15}$ & 368 \\
Device 2 & 0.22 & 48 & $4.8 \times 10^{16}$ & 384 \\
Device 3 & 0.22 & 31 & $3.5 \times 10^{18}$ & 248
\end{tabular}

TABLE II. Results of the fits to the experimental $\mathrm{THz}$ data.

In case of device 3 , the mobility and the electron concentration show excellent agreement with those values, deduced by electrical characterization. At the doping concentration of device 3 , ionized impurity scattering is the main contribution to carrier relaxation in $\mathrm{GaN}$, even at room temperature ${ }^{18}$. Devices 1 and 2 , however, have doping densities that are about two orders of magnitude smaller (see Table I), and ionized impurity scattering can be neglected. Traditional DC measurements on material with $n_{3 D}=5 \times 10^{16} \mathrm{~cm}^{-3}$ would yield a carrier mobility of around $900 \mathrm{~cm}^{2} / \mathrm{Vs}$, from theory ${ }^{19}$ (with $C=0.4$ ) and experimentally observed ${ }^{28}$ for samples without parallel conduction paths at the epi/substrate interface and without scattering processes related to threading dislocations (TDs). On both devices, the $\mathrm{THz}$ measurements yield increased scattering times, which result in nearly identical values of $\mu \approx 375 \mathrm{~cm}^{2} / \mathrm{Vs}$. Hall measurements, however, failed on these samples (see Table I) because of the non-ohmic behavior of the contacts at these low doping densities. The results obtained with $\mathrm{THz}$ electromodulation spectroscopy show that the technique is suited for characterizing even structures with imperfect ohmic contacts. This feature of $\mathrm{THz}$ electromodulation spectroscopy may close a gap that is difficult to access by conventional electronic techniques.

Previous studies that used classical DC characterization showed that TDs significantly reduce mobility ${ }^{20}$. This is not observed in the $\mathrm{THz}$ experiments. The TDDs in devices 1 and 2 differ by about one order of magnitude, but the $\mathrm{THz}$ experiments yield nearly identical mobilities. This observation may be explained by the landscape of the electric potential formed by the negatively charged $\mathrm{TDs}^{20,29}$. In DC characterization, the macroscopic transport of the charge carriers requires passing Coulomb barriers between the individual TDs, which are spaced about $500 \mathrm{~nm}$ and $160 \mathrm{~nm}$ in device 1 and 2, respectively. The root mean square displacement of the carriers by the $\mathrm{THz}$ radiation, however, is only $\langle\ell\rangle=\mu E / \sqrt{8} \pi \nu \approx 20 \mathrm{~nm}$. Thus, the majority of the charge carriers will not suffer scattering during their oscillation at $\mathrm{THz}$ frequencies. In the case of doping concentrations that exceed $10^{18} \mathrm{~cm}^{-3}$, the mobile carriers efficiently screen the potential barriers and the macroscopic transport is less hindered. This explains why conventional characterization and $\mathrm{THz}$ spectroscopy yield nearly identical results, as observed for device 3 .

In conclusion, terahertz electromodulation spectroscopy was applied for studying charge transport in ntype GaN grown on sapphire by MOVPE. All structures investigated reveal Drude transport. The conductivity effective mass of the electrons $m_{c}^{*}$ as well as the relaxation times $\tau$ were determined. At high doping densities, the mobility deduced from $\tau$ and $m_{c}^{*}$ agrees well with the mobility obtained by Hall characterization. At lower densities, where classical techniques are challenging, $\mathrm{THz}$ electromodulation spectroscopy yields reasonable transport parameters.

The research was supported by the German Science Foundation DFG under grants Ke 516/1-2 and Ke 516/61. The authors gratefully acknowledge Coherent Inc. and in particular U. Emmerichs for technical support.

${ }^{1}$ N. Ashcroft and N. Mermin, Solid State Physics (Holt, Rinehart and Winston, 1987)

${ }^{2}$ M. Dressel and M. Scheffler, Ann. Phys. (Leipzig) 15, 535 (2006)

${ }^{3}$ M. van Exter and D. Grischkowsky, Appl. Phys. Lett. 56, 1694 (1990)

${ }^{4}$ M. van Exter and D. Grischkowsky, Phys. Rev. B 41, 12140 (1990) 
${ }^{5}$ W. Zhang, A. K. Azad, and D. Grischkowsky, Appl. Phys. Lett. 82, $2841(2003)$

${ }^{6}$ T.-R. Tsai, S.-J. Chen, C.-F. Chang, S.-H. Hsu, T.-Y. Lin, and C.-C. Chi, Opt. Express 14, 4898 (2006)

${ }^{7}$ H. C. Guo, X. H. Zhang, W. Liu, A. M. Yong, and S. H. Tang, J. Appl. Phys. 106, 063104 (2009)

${ }^{8}$ H. P. Porte, D. Turchinovich, D. G. Cooke, and P. Uhd Jepsen, J. Phys.: Conf. Ser. 193, 012084 (2009)

${ }^{9}$ A. V. Muravjov, D. B. Veksler, V. V. Popov, O. V. Polischuk, N. Pala, X. Hu, R. Gaska, H. Saxena, R. E. Peale, and M. S. Shur, Appl. Phys. Lett. 96, 042105 (2010)

${ }^{10}$ S. Funk, G. Acuna, M. Handloser, and R. Kersting, Opt. Expr. 17, $17450(2009)$

${ }^{11}$ S. G. Engelbrecht, A. J. Reichel, and R. Kersting, J. Appl. Phys. 112, $123704(2012)$

${ }^{12}$ S. G. Engelbrecht, L. De Angelis, M. Tönnies, and R. Kersting, Appl. Phys. A 113, 641 (2013)

${ }^{13}$ S. G. Engelbrecht, M. Prinz, T. R. Arend, and R. Kersting, Appl. Phys. Lett. 105, 012101 (2014)

${ }^{14}$ S. Das Bakshi, J. Sumner, M. J. Kappers, and R. A. Oliver, J. Cryst. Growth 311, 232 (2009)

${ }^{15}$ M. A. Moram, C. S. Ghedia, D. V. S. Rao, J. S. Barnard, Y. Zhang, M. J. Kappers, and C. J. Humphreys, J. Appl. Phys. 106, 073513 (2009)

${ }^{16}$ R. A. Oliver, M. J. Kappers, J. Sumner, R. Datta, and C. J. Humphreys, J. Cryst. Growth 289, 506 (2006)

${ }^{17}$ C. Mavroidis, J. J. Harris, R. B. Jackman, I. Harrison, B. J. Ansell, Z. Bougrioua, and I. Moerman, J. Appl. Phys. 91, 9835 (2002)
${ }^{18}$ C. Mavroidis, J. J. Harris, M. J. Kappers, C. J. Humphreys, and Z. Bougrioua, J. Appl. Phys. 93, 9095 (2003)

${ }^{19}$ D. L. Rode and D. K. Gaskill, Appl. Phys. Lett. 66, 1972 (1995)

${ }^{20}$ N. G. Weimann, L. F. Eastman, D. Doppalapudi, H. M. Ng, and T. D. Moustakas, J. Appl. Phys. 83, 3656 (1998)

${ }^{21}$ L. S. Tan, S. Prakash, K. M. Ng, A. Ramam, S. J. Chua, A. T. S. Wee, and S. L. Lim, Semicond. Sci. Technol. 15, 585 (2000)

${ }^{22}$ J. B. Fortin and T.-M. Lu, Chemical Vapor Deposition Polymerization: The Growth and Properties of Parylene Thin Films (Kluwer Academic Publishers, Dordrecht, 2004)

${ }^{23}$ X. Ai, M. C. Beard, K. P. Knutsen, S. E. Shaheen, G. Rumbles, and R. J. Ellingson, J. Phys. Chem. B 110, 25462 (2006)

${ }^{24}$ M. Dressel and G. Grüner, Electrodynamics of Solids (Cambridge University Press, 2002)

${ }^{25}$ M. Drechsler, D. M. Hofmann, B. K. Meyer, T. Detchprohm H. Amano, and I. Akasaki, Jpn. J. Appl. Phys. 34, L 1178 (1995)

${ }^{26}$ W. Knap, H. Alause, J. M. Bluet, J. Camassel, J. Young, M. Asif Khan, Q. Chen, S. Huant, and M. Shur, Solid State Commun. 99, 195 (1996)

${ }^{27}$ P. Perlin, E. Litwin-Staszewska, B. Suchanek, W. Knap, J. Camassel, T. Suski, R. Piotrzkowski, I. Grzegory, S. Porowski, E. Kaminska, and J. C. Chervin, Appl. Phys. Lett. 68, 1114 (1996)

${ }^{28}$ D. G. Zhao, H. Yang, J. J. Zhu, D. S. Jiang, Z. S. Liu, S. M. Zhang, Y. T. Wang, and J. W. Liang, Appl. Phys. Lett. 89, 112106 (2006)

${ }^{29}$ P. J. Hansen, Y. E. Strausser, A. N. Erickson, E. J. Tarsa, P. Kozodoy, E. G. Brazel, J. P. Ibbetson, U. Mishra, V. Narayanamurti, S. P. Den Baars, and J. S. Speck, Appl. Phys. Lett. 72, 2247 (1998) 